\title{
Endemic goiter-individual risk factors necessitate individual treatment
}

\author{
M. Schneider • T. Welsch • M. Kremer • M. W. Büchler
}

(C) Springer-Verlag 2011

The current issue of Langenbeck's Archives of Surgery coincides with the annual meeting of the German Association of Endocrine Surgeons (CAEK). Therefore, parts of its contents were selected to highlight urgent topics from endocrine surgery. A focus was placed on endemic nodular goiter-the most frequent endocrine disorder requiring surgical intervention.

In an update on state-of-the-art surgical treatment for endemic goiter, Henning Dralle and colleagues compare risks and benefits of total versus subtotal thyroidectomy, and underline the necessity of individualizing the surgical approach according to patient-specific risk factors for disease recurrence and surgical morbidity [1]. Interestingly, as suggested by a clinical study likewise included in this issue [2], long-term quality of life does not seem to be impaired by the extent of thyroid resection. Nonetheless, it is unquestionable that the decision towards a more radical resection approach, while decreasing the risk of recurrence, also increases surgical morbidity - a strong argument in favor of individualized surgical treatment.

Obviously, individualized treatment requires a reliable judgment concerning patient-specific risks for the development of goiter recurrence. For this purpose, a thorough understanding of environmental and genetic risk factors, which individually affect the pathogenesis and recurrence of the disease, is desirable. A scientific review article, contributed by Ralf Paschke, provides an overview on our current understanding of this subject. It delineates how

M. Schneider · T. Welsch • M. Kremer • M. W. Büchler $(\triangle)$ Department of Surgery, University of Heidelberg,

Im Neuenheimer Feld 110,

69120 Heidelberg, Germany

e-mail: markus.buechler@med.uni-heidelberg.de impaired antioxidant defense mechanisms, along with deficient iodine supply and genetic risk factors, concomitantly contribute to mutagenesis in the thyroid gland [3]. A research article by Malkolmes et al. highlights a potential role of platelet-derived growth factors, mitogenic growth factors implicated in tissue remodeling and cellular differentiation, in the development of recurrent goiter [4]. Undoubtedly, more research in this direction is warranted to improve our understanding how growth factors orchestrate thyroid nodular transformations, and which genetic traits are associated with this process.

Collectively, the articles included in this issue, along with the abstracts presented at the current annual meeting of the CAEK, reflect various fruitful attempts at improving long-term success, while at the same time minimizing the risks of surgical treatment for endemic goiter. The development and continuous improvement of intraoperative monitoring systems to avoid recurrent laryngeal nerve palsy [5] is just one example of these ongoing efforts. However, these contributions rightly imply that personalized therapy, an emerging trend not only in cancer treatment, might become a cornerstone for the successful management of this widespread disease.

\section{Conflicts of interest None.}

\section{References}

1. Dralle H, Lorenz K, Machens A (2011) State of the art: surgery for endemic goiter-a plea for individualizing the extent of resection instead of heading for routine total thyroidectomy. Langenbecks Arch Surg 396(8):1137-1143

2. Schmitz-Winnenthal FH, Schimmack S, Lawrence B, Maier U, Heidmann $M$ et al (2011) Quality of life is not influenced by the 
extent of surgery in patients with benign goiter. Langenbecks Arch Surg 396(8):1157-1163

3. Paschke R (2011) Molecular pathogenesis of nodular goiter. Langenbecks Arch Surg 396(8):1127-1136

4. Malkomes P, Oppermann E, Bechstein WO, Holzer K (2011) Significantly high expression of platelet-derived growth factor (PDGF) in benign nodules of the thyroid: relevance in the development of goitre recurrence? Langenbecks Arch Surg 396 (8): $1165-1172$

5. Birkholz T, Saalfrank-Schardt C, Irouschek A, Klein P, Albrecht S et al (2011) Comparison of two electromyographical endotracheal tube systems for intraoperative recurrent laryngeal nerve monitoring: reliability and side effects. Langenbecks Arch Surg 396 (8):1173-1179 